\title{
PROJECTION-BASED INTERPOLATION ALGORITHM OVER TWO-DIMENSIONAL GRIDS WITH TRIANGULAR ELEMENTS
}

\author{
JAKUb RYZNER ${ }^{1}$, ANNA PASZYŃSKA ${ }^{2 *}$ \\ ${ }^{1}$ Faculty of Electrical Engineering, Automatics, Computer Science and Biomedical Engineering, \\ AGH University of Science and Technology, Al. Mickiewicza 30, 30-059 Krakow, Poland \\ ${ }^{2}$ Faculty of Physics, Astronomy and Applied Computer Science, \\ Jagiellonian University, ul. St. Łojasiewicza 11, 30-348 Krakow, Poland \\ *Corresponding author: anna.paszynska@uj.edu.pl
}

\begin{abstract}
In this paper, we employ the projection-based interpolation algorithm for approximation of two-dimensional bitmaps resulting from photographs of multi-phase material samples. The algorithm uses h-adaptive two-dimensional finite element grid with triangular elements. It results in a continuous approximation of material data with Lagrange polynomial, which makes them more suitable for finite element method computations.
\end{abstract}

Key words: Projection-based interpolation, Material science simulations, Continuous material data representation

\section{INTRODUCTION}

The projection-based interpolation was invented by (Demkowicz, 2004), to implement efficient hpadaptive finite element method computations, as described in (Demkowicz, 2006a). The mathematical properties of the method, including the convergence properties, are analyzed in (Demkowicz \& Buffa 2004) and (Demkowicz, 2006b). The method was used in (Gurgul et al., 2013) for continuous approximation of material data using two-dimensional hadaptive finite element method with rectangular elements. The agent-based parallel implementation was also proposed in (Sieniek et al., 2011). The method was extended to three-dimensional grids with hexahedral finite elements in (Goik et al., 2013).

We claim that the triangular meshes are more popular in engineering simulations, and thus we want to show that the PBI method works well with these non-regular grids as well.
In this paper, we propose the version of the method using two-dimensional triangular finite elements. For a non-regular structure of the material data bitmaps, that do not conform with horizontal and vertical lines, we expect to obtain better approximation properties of our method.

We assume that we want to compute the displacements, and thus the solution of the entire problem is needed. In the case when we are computing the strength, it is possible to apply the Saint-Venant principle, and the algorithm described in this paper may be not applicable.

It is possible to extend the code with $h p$ adaptivity, but this is technically more complicated. In general $h$ adaptation works well while recovering the large gradients, and $p$ adaptation work well when we deal with smooth solutions. We compromise by using $h$ adaptation with $p=2$. We do not think that p-adaptation is suitable here since we are not approximating a smooth solution rather we are trying to adapt to jumps in material data. 


\section{PROJECTION-BASED INTERPOLATION WITH H-ADAPTIVE FINITE ELEMENT METHOD}

We employ the following adaptive algorithm (algorithm 1) executed on two-dimensional initial grid constructed with triangular finite elements. The input to the algorithm is a uniform grid as well as the bitmap representing the material data.

How is the problem "solved" over the computational mesh? The projection solver performs the operations in algorithm 2.

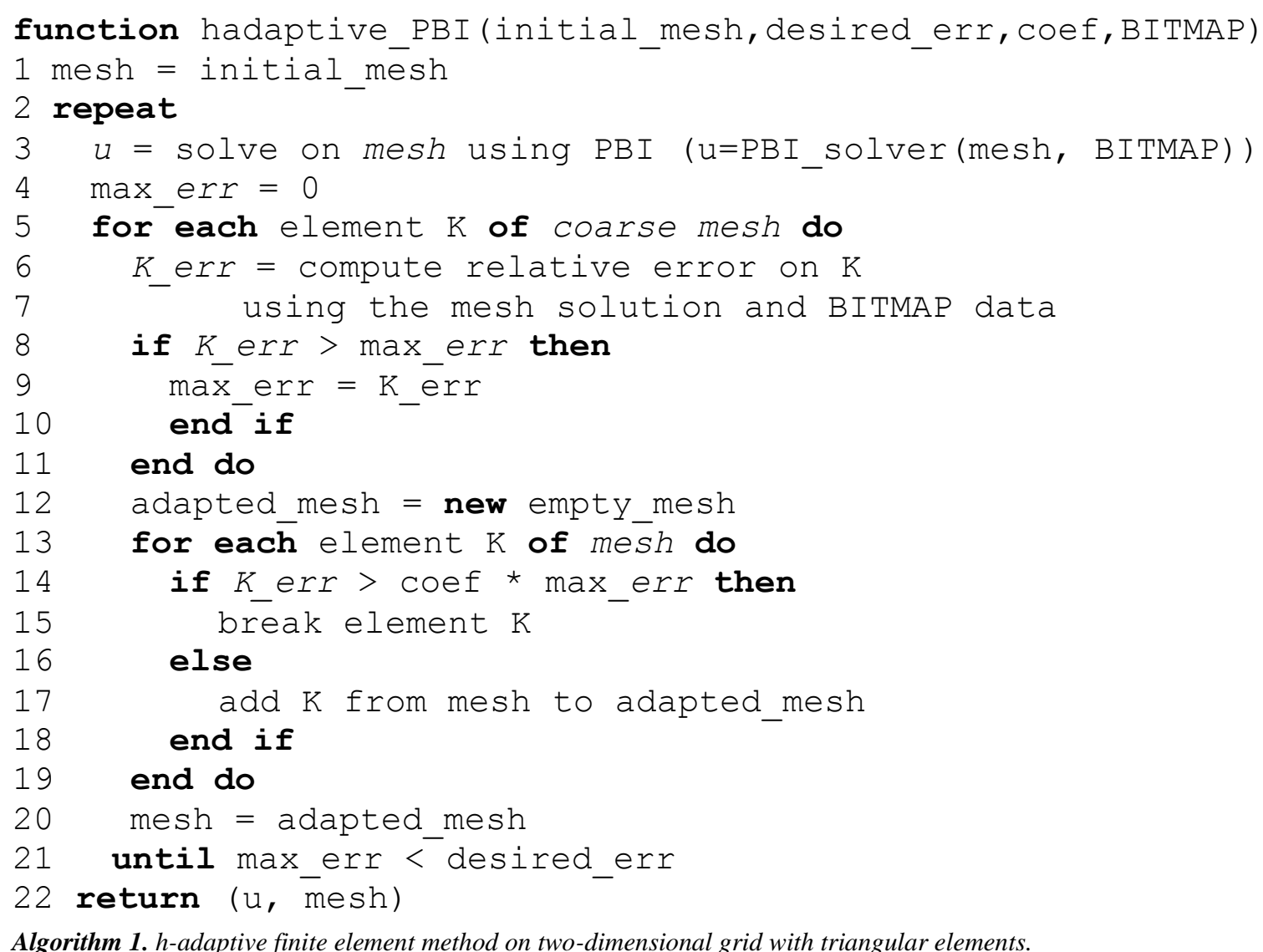

Algorithm 1. h-adaptive finite element method on two-dimensional grid with triangular elements.

\section{function PBI_solver (mesh, BITMAP)}

1 loop through all mesh vertices $v_{i}$

2 //compute the PBI coefficient at vertex $v_{i}$

$3 \quad a_{v_{i}}=\operatorname{BITMAP}\left(v_{i}\right)$

4 end loop

5 loop through all mesh edge nodes $e_{i}$

6 //compute the PBI coefficient at edge $\ell_{i}$

$7 \quad a_{e_{i}}=\frac{\int_{e_{i}} U(x, y) \varphi_{e_{i}}(\mathrm{x}, \mathrm{y}) d x d y}{\int_{e_{i}} \varphi_{e_{i}}(\mathrm{x}, \mathrm{y}) \varphi_{e_{i}}(\mathrm{x}, \mathrm{y}) d x d y}$

8 end loop

9 loop through all mesh interior nodes $I$

10 //compute the PBI coefficient at interior I

$11 a_{I}=\frac{\int_{I} W(\mathrm{x}, \mathrm{y}) \varphi_{I}(\mathrm{x}, \mathrm{y}) d x d y}{\int_{I} \varphi_{I}(\mathrm{x}, \mathrm{y}) \varphi_{I}(\mathrm{x}, \mathrm{y}) d x \mathrm{dy}}$

12 end loop

13 return (u)

Algorithm 2. Projection-based interpolation solver called from line 3 of Algorithm 1. 
The quadratic polynomials are used, thus we have one degree of freedom per vertex, one degree of freedom per edge, and one degree of freedom per element interior. From the point of view of a single triangular element, the algorithm computes the coefficients at all three vertices of the triangular element just by reading them from the bitmap:

$a_{v_{i}}=\operatorname{BITMAP}\left(v_{i}\right) \forall_{i=1, \ldots, 3}$

Next, it minimizes the error in the $L 2$ norm over three edges of the triangle The values read from $\operatorname{BITMAP}\left(v_{i}\right)$ corresponds to pixels as presented in figure 1 . They are within $[0,1]$ where the values close to 0 represent one phase of the material, and the values close to 1 represent the second phase of the material. They are mapped into the Young modulus in a way that one phase maps to $15 \mathrm{GPa}$ and another phase maps to $208 \mathrm{GPa}$ :

$$
\|\overbrace{\left(\text { BITMAT }-\sum_{j=1}^{3} u_{V_{j}}\right)}^{U}-u_{e_{i}}\|_{L 2\left(e_{i}\right)} \rightarrow \min \forall_{i=1, \ldots, 3}
$$

We want to minimize distance from the space span by polynomial $u_{e_{i}}=a_{i} e_{i}$ and the remaining approximant $U$. This is attained by considering $U-u_{e_{i}}$ orthogonal to the space span by $u_{e_{i}}$, which means that the scalar product in L2 norm is 0 there, which literally means:

$$
a_{e_{i}}=\frac{\int_{e_{i}} U(x, y) \varphi_{e_{i}}(\mathbf{x}, \mathbf{y}) d x d y}{\int_{e_{i}} \varphi_{e_{i}}(\mathbf{x}, \mathbf{y}) \varphi_{e_{i}}(\mathbf{x}, \mathbf{y}) d x d y}
$$

since the only basis function in the space span by polynomial $u_{e_{i}}$ is $e_{i}$.

Next, it minimizes the error in the L2 norm over the interior of the triangle:

$$
\|\overbrace{\left(U-\sum_{j=1}^{3} u_{V_{j}}-\sum_{j=1}^{3} u_{0, e_{j}}\right)}^{W}-u_{\mathbf{I}}\|_{L 2\left(f_{i}\right)} \rightarrow \min
$$

We want to minimize the distance from the space span by polynomial $u_{\mathrm{I}}=a_{i} e_{I}$ and the remaining approximant $W$. This is attained by considering
$W-u_{e_{I}}$ orthogonal to the space span by $u_{e_{I}}$, which means that the scalar product in L2 norm is 0 there, which means:

$a_{\mathrm{I}}=\frac{\int_{\mathrm{I}} W(\mathbf{x}, \mathbf{y}) \varphi_{\mathrm{I}}(\mathbf{x}, \mathbf{y}) d x d y}{\int_{\mathrm{I}} \varphi_{\mathrm{I}}(\mathbf{x}, \mathbf{y}) \varphi_{\mathrm{I}}(\mathbf{x}, \mathbf{y}) d x d y}$

since the only basis function in the space span by polynomial $u_{e_{I}}$ is $e_{I}$.

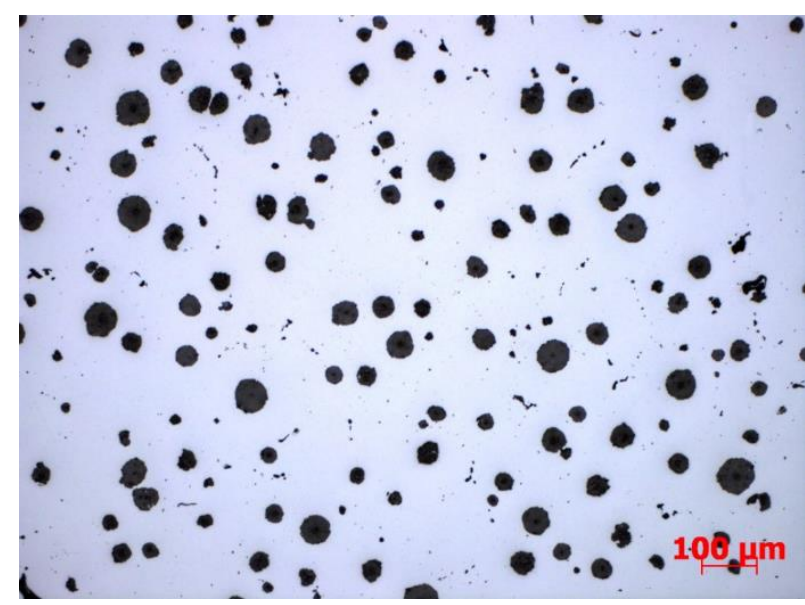

Fig. 1. The original bitmap with photo of the two-phase material.

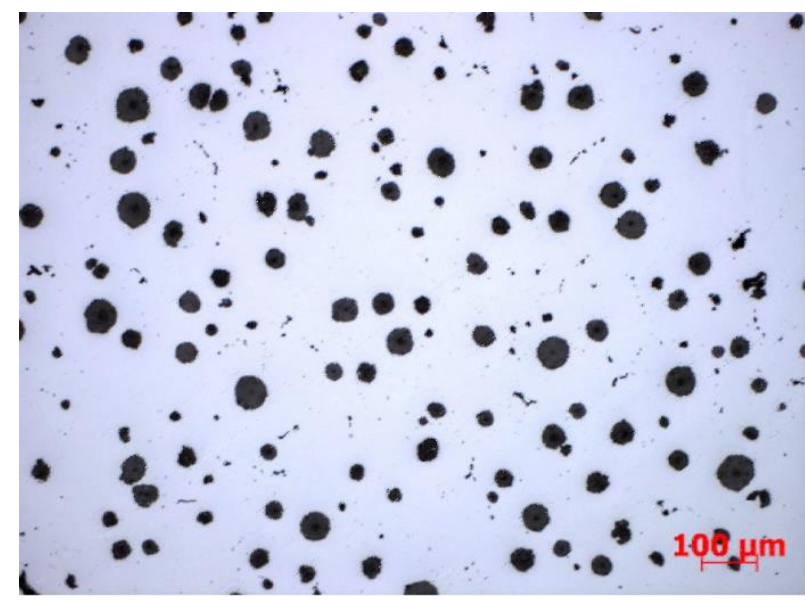

Fig. 2. The continuous approximation of the bitmap.

\section{NUMERICAL RESULTS}

We conclude the paper with numerical results presenting the generation of the continuous approximation of the material data representing the twophase material.

The size of the input mesh is arbitrary, the smaller the better, but we need to be able to catch the material irregularities at the Gauss integration points, so either initial mesh is large enough, or 
while computing the numerical error over an element we can perform the adaptive integration to catch all the irregularities of the bitmap.

We have performed now the linear elasticity simulations of the compressed material. We consider the two-phase material with graphite inclusions under the compressing force.

The properties of the graphite are $E=15 \mathrm{GPa}$ Young modulus and $v=0.3$ Poisson ratio. The properties of the background material are $E=208$ GPa Young modulus and $v=0.3$ Poisson ratio, which represent the steel.
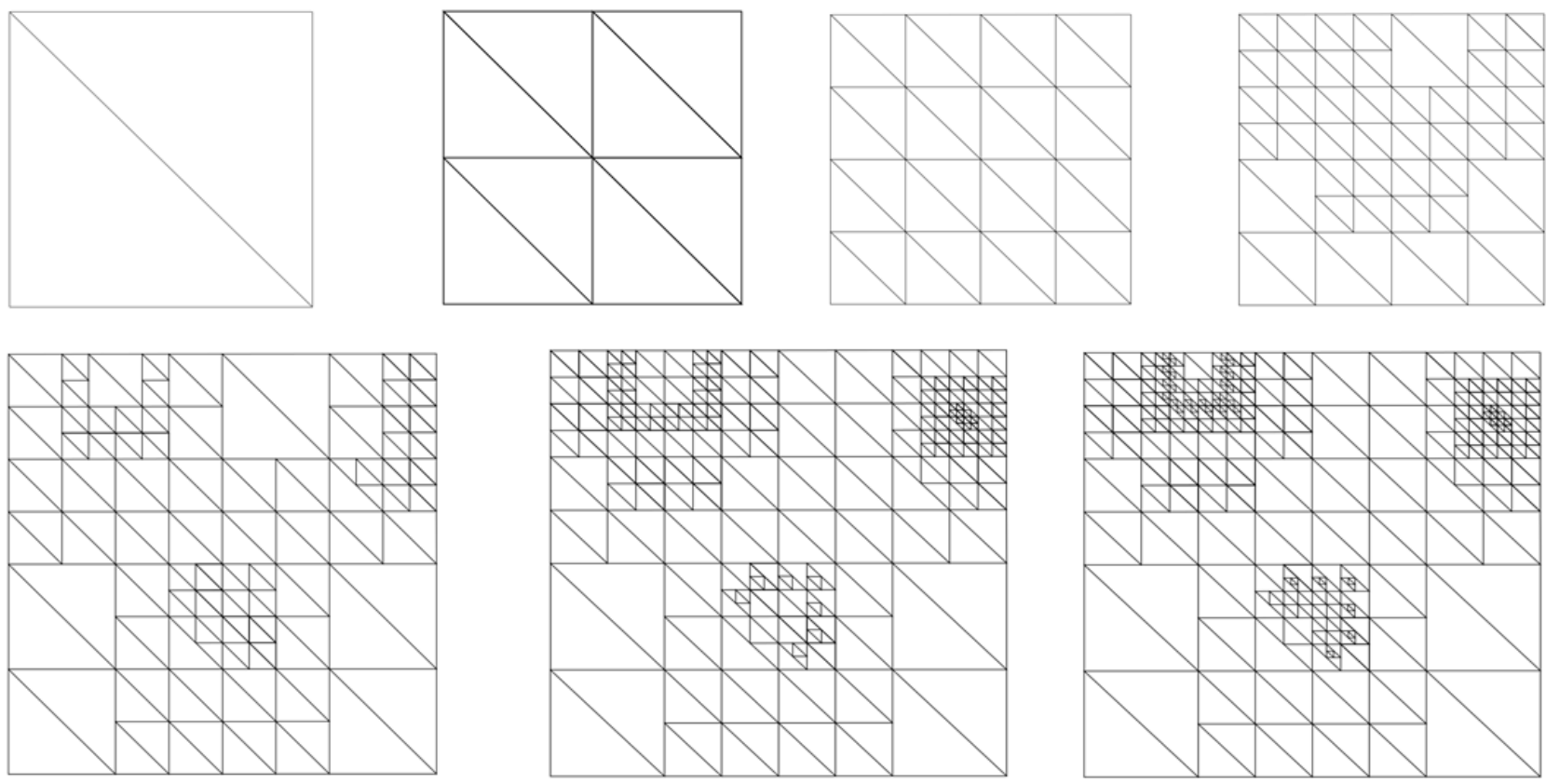

Fig. 3. The fragment of the computational mesh with adaptivities resulting from application of algorithm 1.

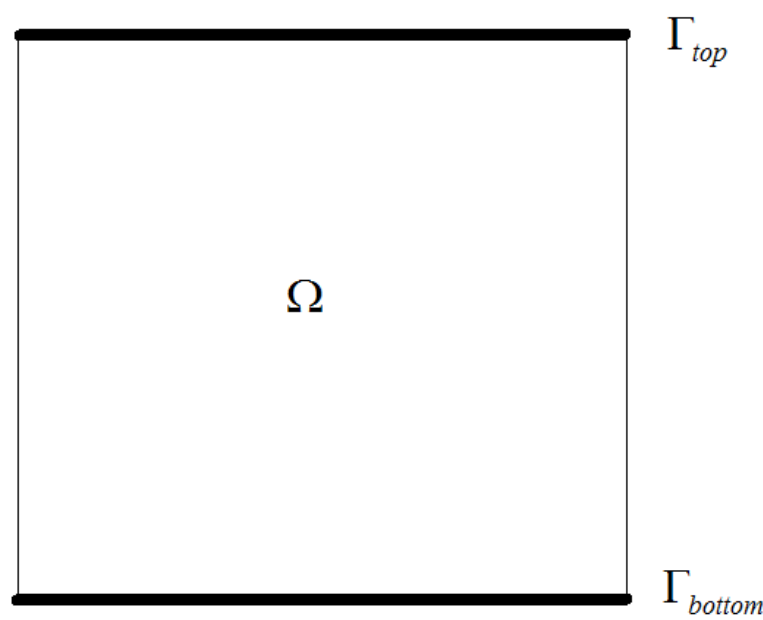

Fig. 4. The continuous approximation of the bitmap.

In our formulation we use displacements, and we model the compression by the following trick. We fix the displacements at the top and at the bottom of the domain, and we define the displacements as $(0$,$1)$ at the top of the boundary, and $(0,1)$ at the bottom of the boundary. Thus, we do not model the force itself, but rather the displacement of the material on the boundary of the domain, following from the compression force. From mathematical point of view, the formulation is stable and well-possed. The problem is elliptic, and on the part of the boundary we have Dirichlet boundary conditions.

We utilize the compressing force:

$$
g_{i}: \Gamma_{D_{i}} \ni x \rightarrow g_{i}(x)=\left\{\begin{array}{lrr}
(0,-1) & \text { for } & x \in \Gamma_{\text {top }} \\
(0,0) & \text { for } & x \in \Omega \backslash\left(\Gamma_{\text {top }} \cup \Gamma_{\text {bottom }}\right) \\
(0,1) & \text { for } & x \in \Gamma_{\text {botom }}
\end{array}\right.
$$

and zero initial stress $\sigma_{i j}^{0}=0$. 
The elliptic computational problem, such as the linear elasticity, where some part of the boundary included the Dirichlet boundary conditions for all the components of the solution derive a unique solution. Here we fix the displacements on the top and bottom of the domain, as expressed by the formula for function gi from (7) the Dirichlet b.c. as described in Appendix. In other words, the function gi is the Dirichlet boundary condition.

We processed the piece of the material as presented in figure 5. Young modulus map in the material is presented in figure 6 . The resulting displacement fields are presented in figures 7 and 8.

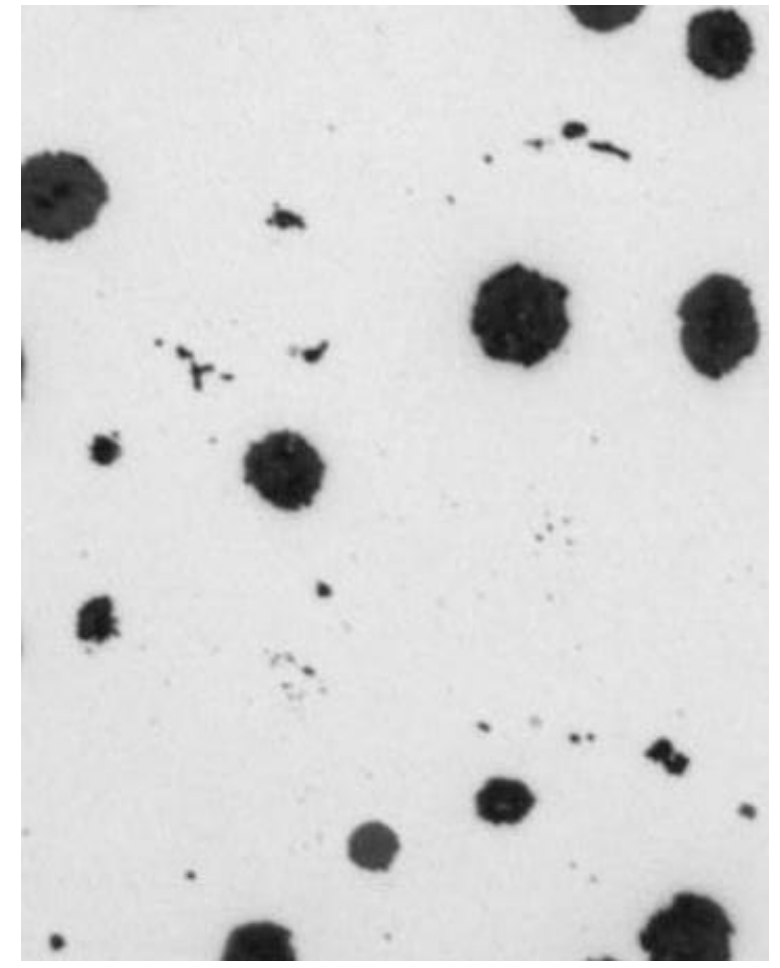

Fig. 5. A Piece of the bitmap used for the compression simulations.
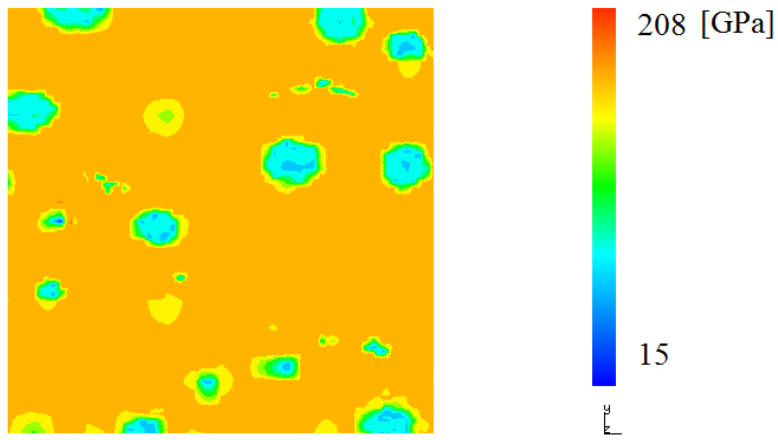

Fig. 6. Young modulus map in the material.

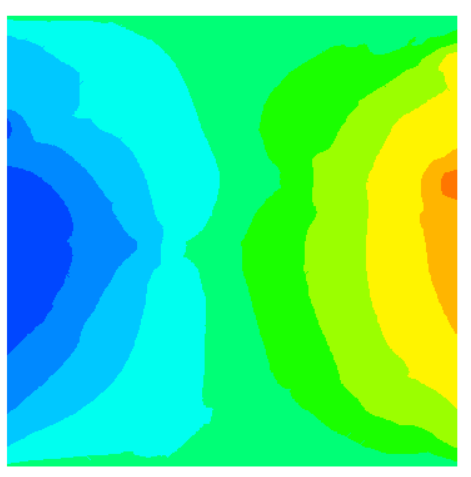

0.5622

Fig. 7. Displacement in the $x$ direction.

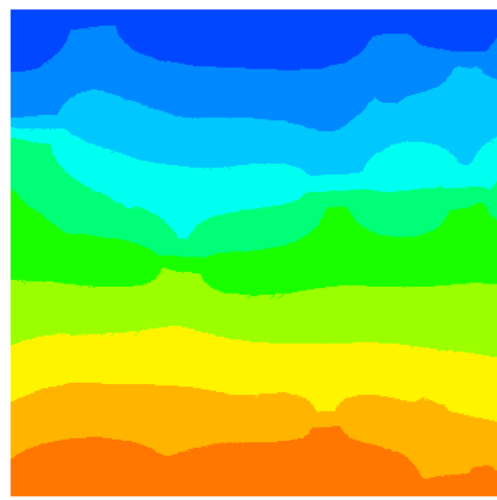

Fig. 8. Displacement in the y direction.

\section{CONCLUSIONS}

The adaptive bitmap compressions with projection-based interpolation algorithm on triangular grids results in the continuous approximation of material data suitable for finite element method computations. We have shown in this paper that the material science data can be successfully approximated with PBI using triangular mesh and subject to finite element method linear elasticity simulations. The continuous approximation of material data removes singularities on the border of materials.

When there are jumps in material coefficients, it is possible to apply the finite element method without continuous approximation of material data obtained by computing some projections, e.g., projection-based approximation as proposed in this paper. However, to converge to the high accuracy of the numerical solution, we need to perform several adaptations of the computational mesh, each time solving a computational problem (linear elasticity in our case). The computational cost of solution of a computational problem in two dimensions is $\mathrm{O}\left((\mathrm{kN})^{3 / 2}\right)$ where $\mathrm{k}$ is the number of equations $(k=2$ in case of linear elasticity), and $N$ is the number of basis functions over the mesh. In three-dimensions (not considered in this paper) this cost grows higher to $\mathrm{O}\left((k N)^{2}\right)$. In other words, the computational cost of 
creating high accuracy adaptive grid based on adaptive solving of a linear elasticity problem requires a sequence of $\mathrm{O}((k N) 3 / 2)$ cost solutions. On the other hand, the projection based algorithm employed in this paper generates a smooth approximation of material data, by employing a similar adaptive process, with the linear computations cost $\mathrm{O}(N)$. We can even employ the adapted computational mesh generated by projection based interpolation algorithm in a linear computational cost and solve the linear elasticity problem with the original distribution of coefficients, resulting in a similar accuracy than the one obtained from the more costly standard adaptive procedure.

\section{APPENDIX A}

We start with classical linear elasticity with $u_{i}$ displacement vector, $u_{i, j}$ displacement gradients, $u_{(\mathrm{i}, j)}$ symmetric part of the displacement gradients $u_{(\mathrm{i}, j)}=$ $\left(u_{i, j}+u_{j, i}\right) / 2$ and $u_{[i, j]}$ skew-symmetric part of the displacement gradients $u_{[i, j]}=\left(u_{i, j}-u_{j, i}\right) / 2$.

We have the property that $u_{i, j}=u_{(\mathrm{i}, j)}+u_{[i, j]}$.

We also introduce $f_{i}$ body force per unit volume.

We introduce $\varepsilon_{i j}$ strain tensor, defined to be the symmetric part of the displacement gradients $\varepsilon_{i j}=$ $\left(u_{i, j}-u_{j, i}\right) / 2$, as well as $\sigma_{i j}$ stress tensor, defined in terms of the generalized Hooke's law: $\sigma_{i j}=c_{i j k l} \varepsilon_{k l}$, with $c_{i j k l}$ elastic coefficients (known for a given material).

We derive first the strong form of the boundaryvalue problem:

Given $\quad f_{i}: \quad \Omega \rightarrow R, \quad g_{i}: \quad \Gamma_{D_{i}} \rightarrow R, \quad h_{i}: \quad \Gamma_{N_{i}} \rightarrow R$, find $u_{i}: \bar{\Omega} \rightarrow R$ such that:

$\sigma_{i j, j}+f_{i}=0$ in $\Omega$

$u_{i}=g_{i}$ in $\Gamma_{D_{i}}$

$\sigma_{i j} n_{j}=h_{i}$ in $\Gamma_{N_{i}}$

where: $\sigma_{i j}=c_{i j k l} \varepsilon_{k l}$.

The weak form is derived in the following way:

Multiply $\sigma_{i j, j}=0$ by $w_{i} \in V^{i}$ and integrate over $\Omega$ to get:

$\int_{\Omega} w_{i} \sigma_{i j, j} d \Omega=0$

$$
-\int_{\Omega} w_{i, j} \sigma_{i j} d \Omega+\int_{\Gamma} w_{i} \sigma_{i j} n_{j} d \Omega=0
$$

We have the property $w_{i, j} \sigma_{i j}=w_{(i, j)} \sigma_{i j}$ since $\sigma_{i j}$ is symmetric tensor, thus:

$-\int_{\Omega} w_{(i, j)} \sigma_{i j} d \Omega+\int_{\Gamma} w_{i} \sigma_{i j} n_{j} d \Omega=0$

We get the weak form of the boundary-value problem:

Given $f_{i:}: \Omega \rightarrow R, \quad g_{i}: \quad \Gamma_{D_{i}} \rightarrow R, \quad h_{i}: \quad \Gamma_{N_{i}} \rightarrow R$, find $u_{i} \in V_{i}$ such that:

$$
\begin{gathered}
\int_{\Omega} w_{(i, j)} \sigma_{i j} d \Omega=\int_{\Omega} w_{i} f_{i} d \Omega+\sum_{i=1}^{n d}\left(\int_{\Gamma_{N_{i}}} w_{i} h_{i} d \Gamma_{N_{i}}\right) \\
\text { for all } w_{i} \in V_{i}
\end{gathered}
$$

We use $\sigma_{i j}=c_{i j k l} \varepsilon_{k l}$ to get:

$$
\int_{\Omega} w_{(i, j)} c_{i j k l} \varepsilon_{k l} d \Omega=\int_{\Omega} w_{i} f_{i} d \Omega+\sum_{i=1}^{n d}\left(\int_{\Gamma_{N_{i}}} w_{i} h_{i} d \Gamma_{N_{i}}\right)
$$

We also use $\varepsilon_{i j}=u_{(\mathrm{i}, j)}$ to get:

$$
\int_{\Omega} w_{(i, j)} c_{i j k l} u_{(k, l)} d \Omega=\int_{\Omega} w_{i} f_{i} d \Omega+\sum_{i=1}^{n d}\left(\int_{\Gamma_{N_{i}}} w_{i} h_{i} d \Gamma_{N_{i}}\right)
$$

Following Hughes (2000) we introduce the following abstract notation:

Given $\mathbf{f}, \mathbf{g}, \mathbf{h}$ find $\mathbf{u} \in \mathbf{V}$, such that:

$$
\mathbf{a}(\mathbf{w}, \mathbf{u})=(\mathbf{w}, \mathbf{f})+(\mathbf{w}, \mathbf{h})_{\Gamma} \text { for all } \mathbf{w} \in \mathbf{V}
$$

$$
\begin{aligned}
& \mathbf{a}(\mathbf{w}, \mathbf{u})=\int_{\Omega} w_{(i, j)} c_{i j k l} u_{(k, l)} d \Omega \\
& (\mathbf{w}, \mathbf{f})=\int_{\Omega} w_{i} f_{i} d \Omega \\
& (\mathbf{w}, \mathbf{h})_{\Gamma}=\sum_{i=1}^{n d}\left(\int_{\Gamma_{N_{i}}} w_{i} h_{i} d \Gamma_{N_{i}}\right)
\end{aligned}
$$

For the implementation purposes we notice that:

$$
\mathbf{a}(\mathbf{w}, \mathbf{u})=\int_{\Omega} \boldsymbol{\varepsilon}(\mathbf{w})^{\mathrm{T}} \operatorname{D} \boldsymbol{\varepsilon}(\mathbf{u}) d \Omega
$$

since $w_{(i, j)} c_{i j k l} u_{(i, j)}=\boldsymbol{\varepsilon}(\mathbf{w})^{\mathbf{T}} \mathbf{D} \boldsymbol{\varepsilon}(\mathbf{u})$ and we have defined:

We integrate by parts: 
$\boldsymbol{\varepsilon}(\mathbf{u})=\left\{\begin{array}{c}u_{1,1} \\ u_{2,2} \\ u_{1,2}+u_{2,1}\end{array}\right\}$

$\boldsymbol{\varepsilon}(\mathbf{w})=\left\{\begin{array}{c}w_{1,1} \\ w_{2,2} \\ w_{1,2}+w_{2,1}\end{array}\right\}$

$D=\left[\begin{array}{ccc}\lambda+2 \mu & \lambda & 0 \\ \lambda & \lambda+2 \mu & 0 \\ 0 & 0 & \mu\end{array}\right]$

where

$\lambda=\frac{v E}{(1+v)(1-2 v)}, \quad \mu=\frac{E}{2(1+v)}$ and $E$ - the Young modulus, $v$ - the Poisson ratio.

\section{APPENDIX B}

In this appendix we describe the modificiations of the hp2d linear elasticity code. The Dirichlet and Naumann boundary conditions have been implemented in the following routines:

Getg - uniform Neumann b.c.

Getmat - material data, in particular Young modulus and Poisson ratio

Getf - the compressing force

Source code of the moodification of the hp2d linear elasticity code.

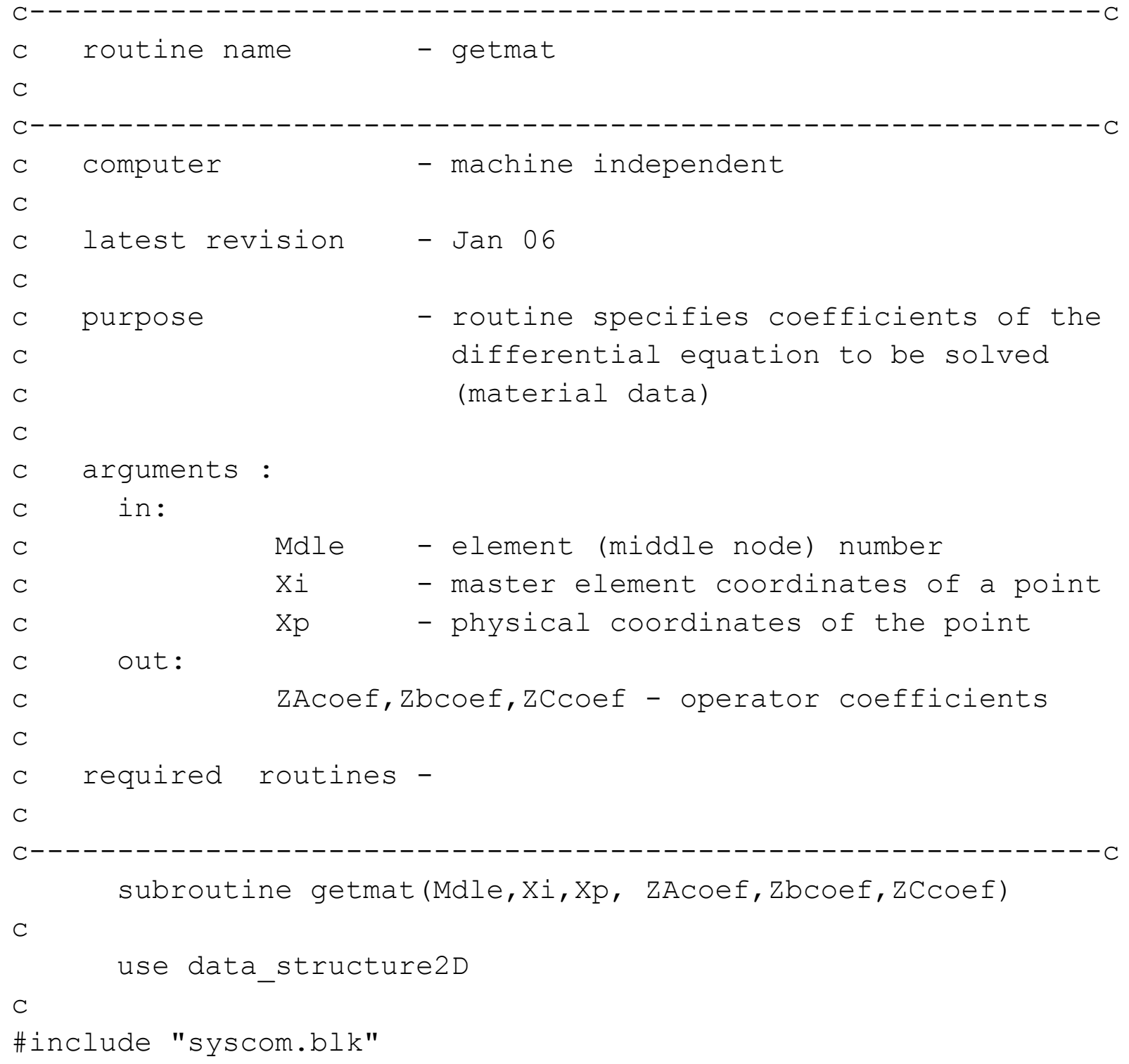


C

dimension Xi(2), Xp (NDIMEN)

dimension ZAcoef (1:MAXEQNS, 1 :MAXEQNS, 2, 2),

Zbcoef ( 1 : MAXEQNS, 1 : MAXEQNS, 2),

ZCcoef ( $1:$ MAXEQNS, $1:$ MAXEQNS )

$\mathrm{C}$

c ...elasticities

dimension ee $(2,2,2,2)$

C

double precision Xval, Xder(2)

C

ZAcoef ( $1:$ MAXEQNS, $1:$ MAXEQNS, $1: 2,1: 2)=$ ZERO

ZBcoef $(1:$ MAXEQNS, $1:$ MAXEQNS, $1: 2)$ = ZERO

ZCcoef $(1:$ MAXEQNS, $1:$ MAXEQNS $)=$ ZERO

C

EYOUNG $=1 . \mathrm{d} 0 ; \mathrm{RNI}=0.3 \mathrm{~d} 0$

call get_bitmap (Xp, Xval, Xder)

if (Xval.1t.0.5d0) then

EYOUNG $=I N V \_E 1 * 1 . d 9$ !graphite

else

EYOUNG $=$ INV_E2*1.d9 !background endif

C

$g=\mathrm{EYOUNG} / 2 \cdot \mathrm{d} 0 /(1 \cdot \mathrm{d} 0+\mathrm{RNI})$

$r I=E Y O U N G * R N I /(1 \cdot d 0+R N I) /(1 \cdot d 0-2 \cdot d 0 * R N I)$

$\mathrm{aux}=2 \cdot d 0 * g+r l$

C

c ...define the tensor of elasticities, see Section 15.2

c in the book

$\mathrm{C}$

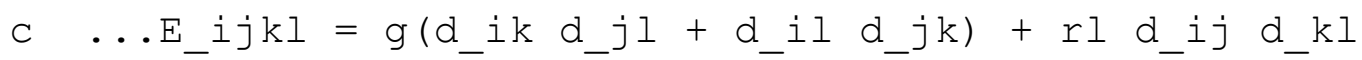

ee $(1: 2,1: 2,1: 2,1: 2)=0 . \bar{d} 0$

ee $(1,1,1,1)=$ aux; ee $(2,2,2,2)=$ aux

ee $(1,1,2,2)=r l$; ee $(2,2,1,1)=r l$

ee $(1,2,1,2)=g ;$ ee $(1,2,2,1)=g$

ee $(2,1,1,2)=g ;$ ee $(2,1,2,1)=g$

C

c ...translate into the format for the PDE's coeeficients

do ivar2 $=1,2$

do $i \operatorname{var} 1=1,2$

do $\mathrm{k}=1,2$

do $1=1,2$

ZAcoef (ivar2, ivar $1, k, 1)=$ ee $(i \operatorname{var} 2, k, i v a r 1,1)$

enddo

enddo 
enddo

enddo

C

c write $\left(*,{ }^{*}\right)$ 'getmat_elas: ZAcoef', ZAcoef

C

return

end

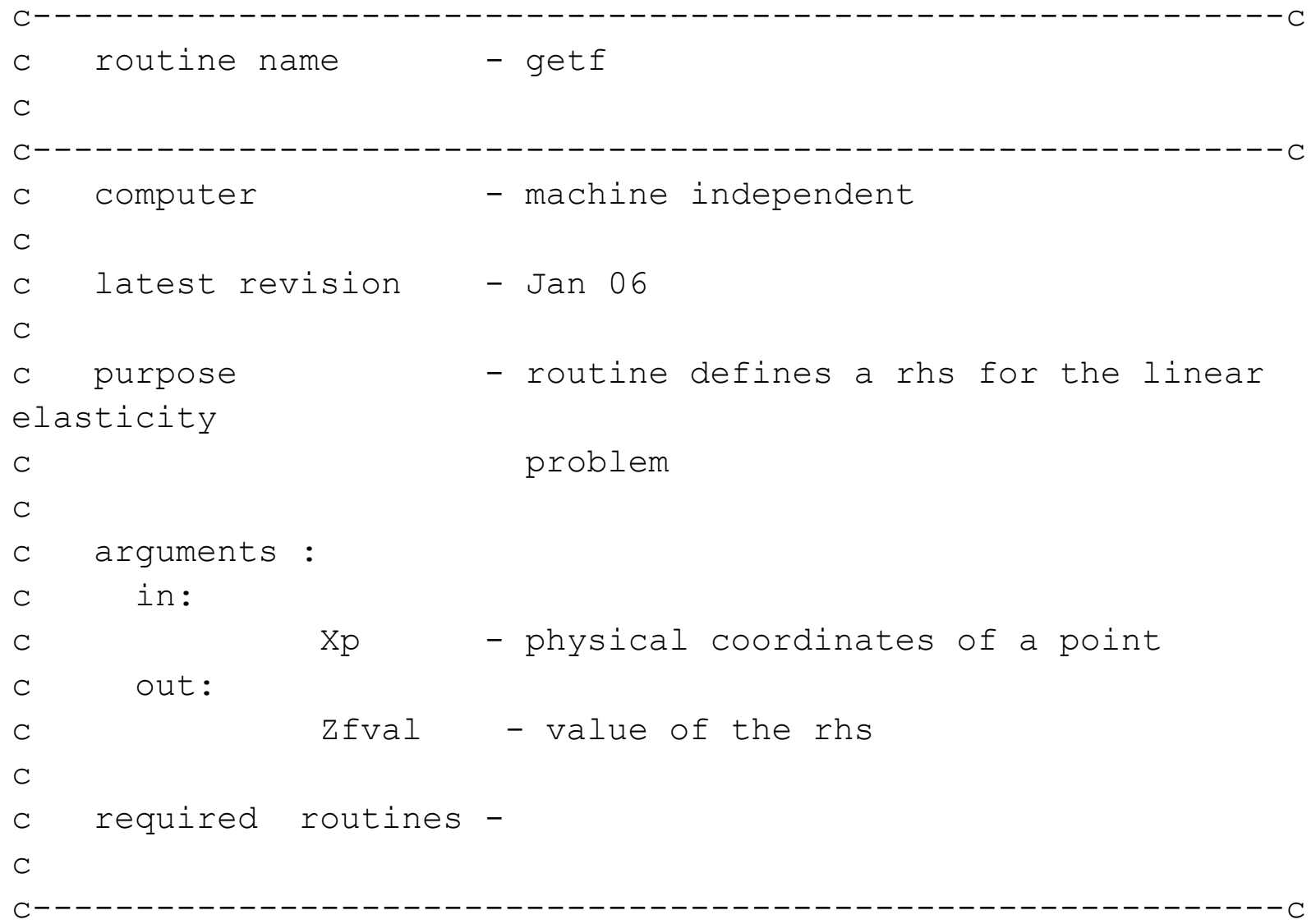

subroutine getf(Mdle,Xi,Xp, Zfval)

C

use data_structure2D

use control

C

\#include "syscom.blk"

C

dimension Xi (2), Xp (NDIMEN), Zfval (MAXEQNS)

dimension zacoef (1:MAXEQNS, 1 :MAXEQNS, 2,2),

zbcoef ( 1 :MAXEQNS, 1 :MAXEQNS, 2 ),

zccoef ( 1 :MAXEQNS, 1 :MAXEQNS)

dimension zval (MAXEQNS), zdvall (MAXEQNS, 2), zdval2 (MAXEQNS, 2, 2)

save nflag getf

data nflag_getf /0/

Zfval $=0 . d 0$

end 


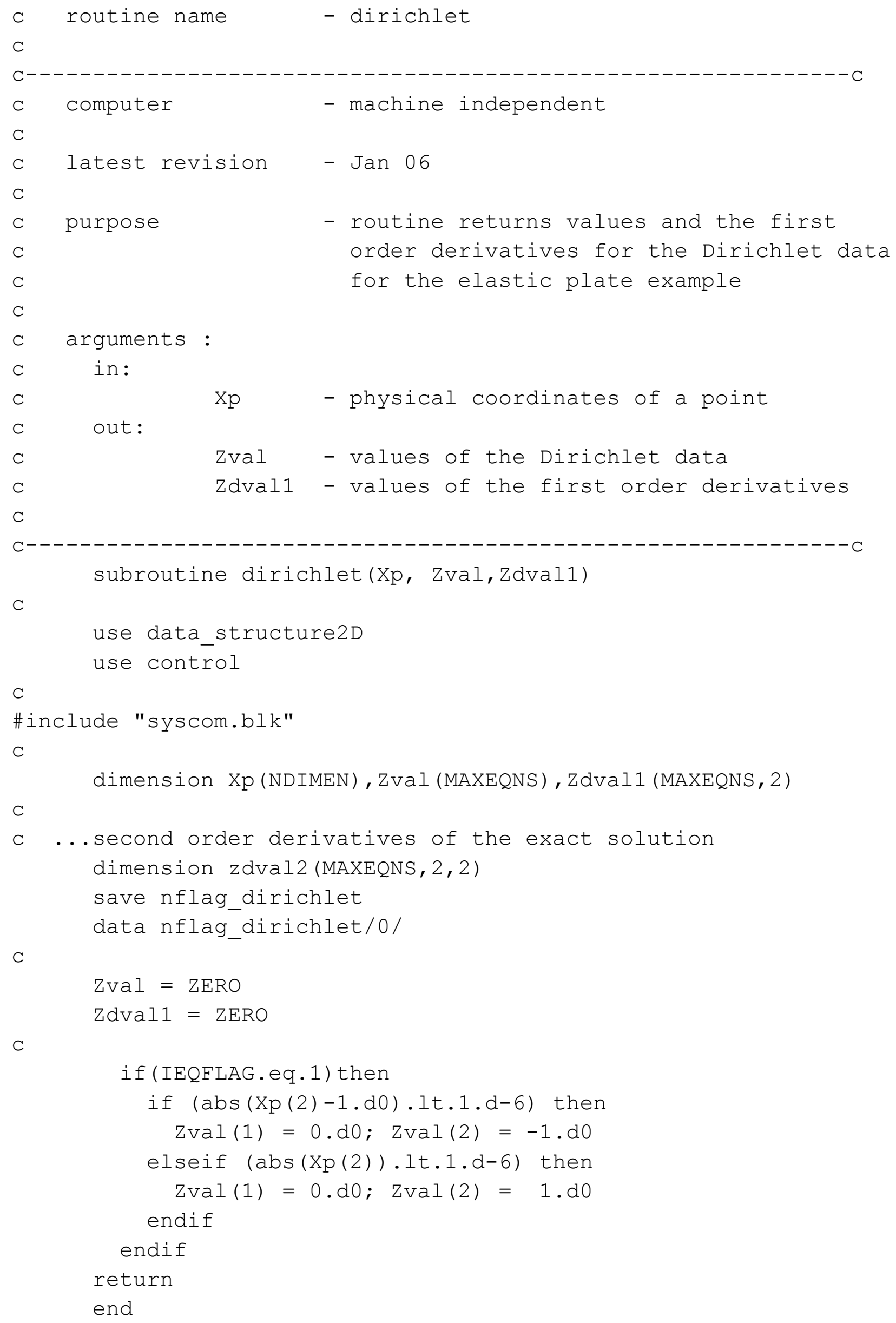




\section{ACKNOWLEDGMENTS}

We would like to acknowledge Maciej Paszyński for help in setup of the hp2d code interface.

\section{REFERENCES}

Demkowicz, L., 2004, Projection-based interpolation, ICES Report 04-03, The University of Texas in Austin.

Demkowicz, L. Buffa, A., 2004, H1, H(curl) and H(div) - conforming projection-based interpolation in three dimensions, ICES-Report 04-24, The University of Texas in Austin.

Demkowicz, L., 2006a, Computing With Hp-adaptive Finite Elements, Chapman \& Hall CRC Press.

Demkowicz, L., 2006b, Polynomial exact sequences and projection - based interpolation with application to Maxwell equations, ICES-Report 06-12, The University of Texas in Austin.

Goik, D., Sieniek, M., Paszynski, M., Madej, Ł., 2013, Employing an Adaptive Projection-based Interpolation to Prepare Discontinuous 3D Material Data for Finite Element Analysis, Procedia Computer Science, 18, 1535-1544.

Gurgul, P., Sieniek, M., Magiera, K., Skotniczny, M., 2013, Application of multi-agent paradigm to hp-adaptive projection-based interpolation operator, Journal of Computational Science, 4(3), 164-169.

Hughes, T.J.R., 2000, The finite element method: Linear statics and dynamics finite element method analysis, Dover Publications.

Sieniek, M., Gurgul, P., Skotniczny, M., Magiera, K., Paszynski, M., 2011, Agent-oriented image processing with the hpadaptive projection-based interpolation operator, Procedia Computer Science, 4, 1844-1853.

\section{ALGORYTM INTERPOLACJI BAZUJACEJ NA PROJEKCJI DLA DWU-WYMIAROWYCH SIATEK OBLICZENIOWYCH Z ELEMENTAMI TRÓJKĄTNYMI}

\section{Streszczenie}

Artykuł przedstawia zastosowanie algorytmu interpolacji bazującej na projekcji do aproksymacji dwuwymiarowych bitmap reprezentujących zdjęcia struktury wielo-fazowych materiałów. Algorytm ten używa $h$-adaptacyjnej dwuwymiarowej metody elementów skończonych z elementami trójkątnymi. W wyniku jego zastosowania dostajemy ciągłą aproksymację materiału $\mathrm{z}$ wykorzystaniem wielomianów Lagrange'a rozpiętych na siatce obliczeniowej, na której można następnie przeprowadzić obliczenia metodą elementów skończonych.

Received: December 2, 2018 Received in a revised form: April 23, 2019. Accepted: June 14, 2019. 